\title{
Inelastic scattering and heating in a molecular spin pump
}

\author{
Jonas Fransson ${ }^{1}$ and Michael Galperin ${ }^{2}$ \\ ${ }^{1}$ Department of Physics and Materials Science, Uppsala University, Box 530, SE-751 21 Uppsala, Sweden \\ ${ }^{2}$ Department of Chemistry 83 Biochemistry, University of California at San Diego, La Jolla CA 92093, USA
}

(Dated: October 31, 2018)

\begin{abstract}
We consider a model for a spin field-effect molecular transistor, where a directed pure spin current is controlled by an external electric field. Inelastic scattering effects of such molecular device are discussed within a framework of full counting statistics for a multi-level molecular system. We propose that the heating of the molecular junction can be controlled by external electric and magnetic fields. Characteristic features of the model are demonstrated by numerical calculations.
\end{abstract}

PACS numbers: 85.65.+h 85.75.Hh 71.38.-k 73.20.Hb

\section{INTRODUCTION}

Fast development of experimental techniques allowing for miniaturization of electronic devices led to renewed interest in theoretical research in the area of quantum transport. In particular in molecular electronics community focus of the research was shifting from elastic (Landauer) to inelastic charge transport through molecular junction, to its noise characteristics and heating. Spin flux as an alternative to charge current in electronic devices, where magnetic field provides an additional (to bias) mean of control, is studied by spintronics $\frac{1,2}{1}$ Quantum ratchets ${ }^{3,4}$ and electric potential ${ }^{5-\underline{7}}$ were proposed as additional controls of the spin flux. Schemes for optical control of a spin trapped in quantum dots were reported in the literature $\underline{\underline{8}-13}$ Recently a combination of spintronics with molecular electronics started to reveal itself as molecular spintronics. $\stackrel{14}{-17}$ Theoretical schemes for spin pumps were considered in Refs. 18 20, and inelastic effects of spin transport through the junctions were reported for spin valves 21 and for tunneling through a junction with embedded spin $\frac{22}{2}$ Shot noise of spin current was considered in Ref. 23.

Here we present a model for a molecular junction, consisting of a molecule between two normal metallic contacts, where pure spin current is controlled by an external electric field. The spin field-effect transistor is a generalization of a spin pump introduced in Ref. 18. Directed spin current seems to be a more convenient element for molecular spintronics device. As in the case of the spin pump, only pure spin current participates in the transport. We consider inelastic effects of the spin current revealed in the transport properties of the device. This consideration takes place as a part of a general approach of full counting statistics for multi-level molecular systems. In particular, we discuss current and zero frequency noise of the junction.

Flexibility of molecules, and as a result wellpronounced inelastic features in transport properties of molecular junctions, makes heating of a device an important issue in molecular electronics. Spin field-effect transistors (FET) inherit this problem of the usual FETs, since spin current is also caused by electrons cross- ing molecule-contact interface which results in heating molecular device. Within the model we discuss spin current through molecular junction, and propose external electric and magnetic fields as possible controls capable of tuning molecular structure to diminish heating of the device.

Section [II presents a model and outlines the method. Section 【II is devoted to full counting statistics of multilevel molecular systems. We describe general approach and introduce junction characteristics - spin and charge current and noise. Section IV is devoted to heating in spin field-effect transistors. In section $\mathbf{V}$ we present results of numerical simulations. Section $\nabla \mathbf{V I}$ concludes.

\section{MODEL}

We consider extension of the spin pump proposed in Ref. 18 to the junction situation. The spin pump of Ref. 18 is a model of a two-level system coupled to one electrode. Application of magnetic field generates spin current in the contact due to spin-flip process taking place at the molecule. We propose a generalization where the spin flux is generated across the junction (spin current between two contacts). Note that the spin flux in our model is optically controlled, i.e. external electric rather than magnetic field controls spin flux. The junction is composed of two molecules (represented by single levels 1 and 2 coupled to molecular vibrations $\omega_{1}$ and $\left.\omega_{2}\right)$ attached to left $L$ and right $R$ normal metal electrodes, respectively. Each molecule is subject to a $d c$ and $a c$ pair of magnetic fields $B_{i}^{(d c)}$ and $B_{i}^{(a c)}(i=1,2)$. Note that level dependent g-factors in nanowire quantum

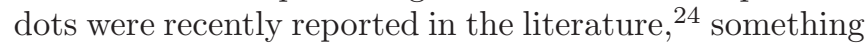
that, here, is represented by the site dependent field. The molecules are weakly coupled by external source of light of particular frequency $\omega_{E}$. The light can be used as a handle to switch on and off spin transport through the junction. The Hamiltonian of the system is

$$
\hat{H}=\hat{H}_{0}+\hat{V},
$$


where

$$
\begin{aligned}
\hat{H}_{0}= & \sum_{k \in L, R ; \sigma} \varepsilon_{k} \hat{n}_{k \sigma}+\sum_{i=1,2}\left(\sum_{\sigma}\left[\varepsilon_{i \sigma}+M_{i}\left(\hat{a}_{i}+\hat{a}_{i}^{\dagger}\right)\right] \hat{n}_{i \sigma}\right. \\
& \left.+\omega_{i} \hat{a}_{i}^{\dagger} \hat{a}_{i}-g \mu_{B} B_{i}^{(a c)}\left(\hat{d}_{i \uparrow}^{\dagger} \hat{d}_{i \downarrow} e^{i \omega_{i}^{(B)} t}+H . c .\right)\right) \\
& +\sum_{k \in L ; \sigma}\left(V_{1 k} \hat{d}_{1 \sigma}^{\dagger} \hat{c}_{k \sigma}+H . c .\right) \\
& +\sum_{k \in R ; \sigma}\left(V_{2 k} \hat{d}_{2 \sigma}^{\dagger} \hat{c}_{k \sigma}+H . c .\right) \\
\hat{V}= & \left(V_{E} e^{-i \omega_{E} t}+V_{E}^{*} e^{i \omega_{E} t}\right) \sum_{\sigma}\left(\hat{d}_{1 \sigma}^{\dagger} \hat{d}_{2 \sigma}+H . c .\right)
\end{aligned}
$$

where $d_{i \sigma}^{\dagger}\left(\hat{d}_{i \sigma}\right)$ and $\hat{c}_{k \sigma}^{\dagger}\left(\hat{c}_{k \sigma}\right)$ are creation (annihilation) operators for corresponding state in the molecule(s) and in the contact(s), $\hat{n}_{i \sigma}=\hat{d}_{i \sigma}^{\dagger} \hat{d}_{i \sigma}$ and $\hat{n}_{k \sigma}=\hat{c}_{k \sigma}^{\dagger} \hat{c}_{k \sigma}, \hat{a}_{i}^{\dagger}\left(\hat{a}_{i}\right)$ are creation (annihilation) operator for vibration quanta of molecule $i, \sigma= \pm 1$ is direction of spin projection, and where

$$
\varepsilon_{i \sigma}=\varepsilon_{i}-\frac{\sigma}{2} g \mu_{B} B_{i}^{(d c)} .
$$

We start by transforming the Hamiltonian (1) into the rotating frames of the magnetic field $\underline{20,25}$

$$
\begin{aligned}
\hat{H} \rightarrow \hat{\bar{H}} & =i\left(\frac{\partial}{\partial t} e^{\hat{S}_{B}(t)}\right) e^{-\hat{S}_{B}(t)}+e^{\hat{S}_{B}(t)} \hat{H} e^{-\hat{S}_{B}(t)}, \\
S_{B}(t)= & -i \frac{\omega_{1}^{(B)}}{2} t \sum_{\sigma} \sigma\left(\hat{n}_{1 \sigma}+\sum_{k \in L} \hat{n}_{k \sigma}\right) \\
& -i \frac{\omega_{2}^{(B)}}{2} t \sum_{\sigma} \sigma\left(\hat{n}_{2 \sigma}+\sum_{k \in R} \hat{n}_{k \sigma}\right) .
\end{aligned}
$$

This transformation eliminates the time-dependence from the ac magnetic field terms, shifts the positions of molecular levels, and induces spin biases in the contacts, according to

$$
\begin{aligned}
& \bar{\varepsilon}_{i \sigma}=\varepsilon_{i \sigma}+\sigma \omega_{i}^{(B)} / 2, \\
& \bar{\varepsilon}_{k \sigma}= \begin{cases}\varepsilon_{k}+\sigma \omega_{1}^{(B)} / 2 & k \in L, \\
\varepsilon_{k}+\sigma \omega_{2}^{(B)} / 2 & k \in R .\end{cases}
\end{aligned}
$$

The perturbation (3) takes the form

$$
\begin{aligned}
\hat{\bar{V}} & =\left(V_{E} e^{-i \omega_{E} t}+V_{E}^{*} e^{i \omega_{E} t}\right) \\
& \times \sum_{\sigma}\left(\hat{d}_{1 \sigma}^{\dagger} \hat{d}_{2 \sigma} e^{-i \sigma\left(\omega_{1}^{(B)}-\omega_{2}^{(B)}\right) t / 2}+\text { H.c. }\right)
\end{aligned}
$$

In what follows we will put $\omega_{1}^{(B)}=-\omega_{2}^{(B)} \equiv \omega_{B}$ to create a spin bias across the junction (see Fig. 1 for a sketch). Similarly, we put $g \mu_{B} B_{1}^{(d c)}=-g \mu_{B} B_{2}^{(d c)} \equiv \omega_{0}$. The

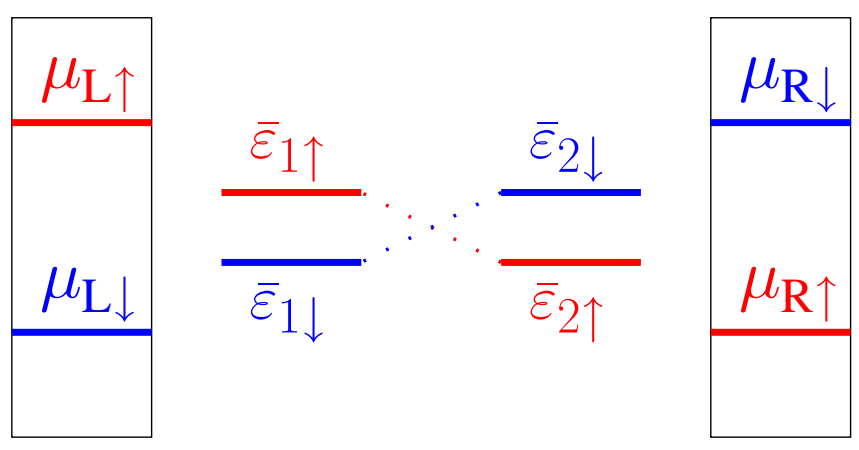

FIG. 1: (Color online) Sketch of an optically controlled spin field-effect transistor.

interaction with the external optical field is taken into account within perturbation theory. Expansion of the evolution operator on the Keldysh contour up to second order in (9) in the rotating-wave approximation (RWA) leads to electronic self-energy due to interaction with external optical field in the form (see Appendix $\mathrm{A}$ for details)

$$
\begin{aligned}
& \Sigma_{\sigma, \sigma^{\prime}}^{(E)}\left(\tau, \tau^{\prime}\right)=\delta_{\sigma, \sigma^{\prime}} 2\left|V_{E}\right|^{2} \cos \omega_{E}\left(t-t^{\prime}\right) \\
& \times\left[\begin{array}{cc}
G_{2 \sigma, 2 \sigma}\left(\tau, \tau^{\prime}\right) e^{-i \sigma \omega_{B}\left(t-t^{\prime}\right)} & 0 \\
0 & G_{1 \sigma, 1 \sigma}\left(\tau, \tau^{\prime}\right) e^{i \sigma \omega_{B}\left(t-t^{\prime}\right)}
\end{array}\right] .
\end{aligned}
$$

As a result of the transformation to the rotating frames and the RWA we obtain a time-independent (steadystate) description.

The coupling between electronic and vibrational degrees of freedom is treated below within two different approaches. The transport through the junction is considered in a basis obtained by a small polaron transformation $\underline{26}$

$$
\begin{aligned}
& \hat{H} \rightarrow \hat{\bar{H}}=e^{\hat{S}_{v}} \hat{H} e^{-\hat{S}_{v}} \\
& \hat{S}_{v}=\sum_{i=1,2 ; \sigma} \frac{M_{i}}{\omega_{i}}\left(\hat{a}_{i}^{\dagger}-\hat{a}_{i}\right) \hat{n}_{i \sigma} .
\end{aligned}
$$

This transformation decouples the electronic and vibrational degrees of freedom on the molecule and dresses the molecular fermion operators

$$
\hat{d}_{i \sigma} \rightarrow \hat{d}_{i \sigma} \hat{X}_{i} \quad \hat{X}_{i}=\exp \left[-\frac{M_{i}}{\omega_{i}}\left(\hat{a}_{i}^{\dagger}-\hat{a}_{i}\right)\right]
$$

We take the vibrational shift operators $\hat{X}$ into account within the usual Born-Oppenheimer-like approximation, which allows us to introduce the Franck-Condon factors 27 multiplying the electronic GF

$$
\begin{aligned}
& G_{i \sigma, j \sigma^{\prime}}\left(\tau, \tau^{\prime}\right) \equiv-i\left\langle T_{c} \hat{d}_{i \sigma}(\tau) \hat{X}_{i}(\tau) \hat{d}_{j \sigma^{\prime}}\left(\tau^{\prime}\right) \hat{X}_{j}\left(\tau^{\prime}\right)\right\rangle \\
& \approx-i\left\langle T_{c} \hat{d}_{i \sigma}(\tau) \hat{d}_{j \sigma^{\prime}}^{\dagger}\left(\tau^{\prime}\right)\right\rangle\left\langle T_{c} \hat{X}_{i}(\tau) \hat{X}_{j}^{\dagger}\left(\tau^{\prime}\right)\right\rangle
\end{aligned}
$$

In the description of the junction heating, we utilize the Born approximation 28 instead, which allows us to 
keep the consideration simple. As usual we implement a non-crossing approximation, i.e. diagrams for electron transfer and interaction with vibrations do not cross (the processes do not happen simultaneously) $\stackrel{26}{\underline{2}}$ In this case Green function is obtained from the Dyson equation

$$
\begin{aligned}
G_{i \sigma, j \sigma^{\prime}}\left(\tau, \tau^{\prime}\right) & =G_{i \sigma, j \sigma^{\prime}}^{(0)}\left(\tau, \tau^{\prime}\right) \\
& +\sum_{m_{1}, m_{2}} \sum_{\sigma_{1}, \sigma_{2}} \int_{c} d \tau_{1} \int_{c} d \tau_{2} G_{i \sigma, m_{1} \sigma_{1}}^{(0)}\left(\tau, \tau_{1}\right) \\
& \times \Sigma_{m_{1} \sigma_{1}, m_{2} \sigma_{2}}^{(p h)}\left(\tau_{1}, \tau_{2}\right) G_{m_{2} \sigma_{2}, j \sigma^{\prime}}\left(\tau_{2}, \tau^{\prime}\right)
\end{aligned}
$$

where $G^{(0)}$ is Green function in the absence of electronphonon interaction $\left(M_{1,2}=0\right)$, and $\Sigma^{(p h)}$ is self-energy due to electron-phonon interaction

$$
\Sigma_{i \sigma, j \sigma^{\prime}}^{(p h)}\left(\tau, \tau^{\prime}\right)=i \delta_{i, j}\left|M_{i}\right|^{2} D_{i}\left(\tau, \tau^{\prime}\right) G_{i \sigma, i \sigma^{\prime}}\left(\tau, \tau^{\prime}\right)
$$

Here $D_{i}\left(\tau, \tau^{\prime}\right) \equiv-i\left\langle T_{c} \hat{a}_{i}(\tau) \hat{a}_{i}^{\dagger}\left(\tau^{\prime}\right)\right\rangle$ is phonon Green function. We treat it within a quasi-particle approximation ${ }^{29}$

\section{FULL COUNTING STATISTICS}

The theoretical concept of full counting statistics was originally proposed by Levitov and Lesovik $\stackrel{30,31}{\text { The ap- }}$ proach was applied to the non-equilibrium Anderson impurity model in Ref. 33. Measurements of shot noise in molecular junction ${ }^{34}$ prove possibility of experimental observation of moments beyond average current. This together with the flexibility of molecules, i.e. importance of inelastic effects in transport through molecular junctions, recently caused several theoretical investigations devoted to study of resonant level coupled to single vibration model $\underline{35-37}$

Here we discuss a generalization of the result derived by Gogolin and Komnik ${ }^{33}$ to a multilevel situation, and apply the expression to calculate the first (current) and second (noise) cumulants of the distribution. Following the derivation of Ref. 33 for the case of multilevel molecule we obtain an expression for the derivative of the adiabatic potential in the form ${ }^{38}$

$$
\begin{aligned}
& \frac{\partial}{\partial \lambda_{K}^{-}} \mathcal{U}\left(\lambda_{K}^{-}, \lambda_{K}^{+}\right)=-\frac{1}{2} \int_{-\infty}^{+\infty} \frac{d E}{2 \pi} \operatorname{Tr}\left\{\boldsymbol{\Sigma}_{K}^{<}(E) e^{i \lambda_{K}} \mathbf{G}_{\lambda}^{>}-\mathbf{G}_{\lambda}^{>} \boldsymbol{\Sigma}_{K}^{<}(E) e^{-i \lambda_{K}}\right\} \equiv-\frac{1}{2} \int_{-\infty}^{+\infty} \frac{d E}{2 \pi} \\
& \operatorname{Tr}\left\{\boldsymbol{\Sigma}_{K}^{<}(E) e^{i \lambda_{K}}\left[\left(E-\mathbf{H}_{0}-\boldsymbol{\Sigma}^{--}(E)\right)\left[\boldsymbol{\Sigma}_{\lambda}^{>}(E)\right]^{-1}\left(E-\mathbf{H}_{0}+\boldsymbol{\Sigma}^{++}(E)\right)+\boldsymbol{\Sigma}_{\lambda}^{<}(E)\right]^{-1}\right. \\
& \left.\quad-\left[\left(E-\mathbf{H}_{0}+\boldsymbol{\Sigma}^{++}(E)\right)\left[\boldsymbol{\Sigma}_{\lambda}^{<}(E)\right]^{-1}\left(E-\mathbf{H}_{0}-\boldsymbol{\Sigma}^{--}(E)\right)+\boldsymbol{\Sigma}_{\lambda}^{>}(E)\right]^{-1} \boldsymbol{\Sigma}_{K}^{>} e^{-i \lambda_{K}}\right\} .
\end{aligned}
$$

Here, $\lambda_{K}^{-}\left(\lambda_{K}^{+}\right)$is a counting field for the interface between the molecule and contact $K$ on the forward (backward) branch of the Keldysh contour, $\lambda_{K} \equiv\left(\lambda_{K}^{-}-\lambda_{K}^{+}\right) / 2$. '--', ' ++ ', ' $<$ ', and ' $>$ ' are causal, anti-causal, lesser, and greater projections. The trace runs over molecular degrees of freedom. The electronic self-energy due to the coupling to contact $K$ is denoted by $\boldsymbol{\Sigma}_{K}$, and $\boldsymbol{\Sigma}_{\lambda}$ is the total electronic self-energy dressed with the counting field $\lambda$. In particular, within the non-crossing approximation $\underline{26}$

$$
\boldsymbol{\Sigma}_{\lambda}^{>,<}(E)=\sum_{K} \boldsymbol{\Sigma}_{K}^{>,<}(E) e^{\mp i \lambda_{K}}+\boldsymbol{\Sigma}_{i n t, \lambda}^{>,<}(E),
$$

where $\boldsymbol{\Sigma}_{i n t, \lambda}^{>,<}$is the electronic self-energy due to interactions dressed with the counting field $\lambda$.

It seems difficult to obtain an expression for the logarithm of the generating function (integral of (17) over $\left.\lambda_{K}^{-}\right)$in the multi-level case. However, Eq. (17) itself can be used to calculate cumulants. The time-averaged charge cumulant of order $n$ due to charge transport through interfaces $\left\{K_{j}\right\}(j=\{1,2, \ldots, n\})$ is

$$
\begin{aligned}
& \frac{\left\langle\delta^{n} q\right\rangle_{K_{n}, \ldots, K_{1}}}{T}= \\
& \quad-\left.i \frac{\partial}{\partial\left(i \lambda_{K_{n}}\right)} \cdots \frac{\partial}{\partial\left(i \lambda_{K_{1}}\right)} \mathcal{U}(\{\lambda\},-\{\lambda\})\right|_{\{\lambda\}=0} .
\end{aligned}
$$

Here and below $e=\hbar=1$.

First cumulant yields the well-know expression for steady-state current ${ }^{39.40}$

$$
\begin{aligned}
I_{K} & =-\left.i \frac{\partial}{\partial\left(i \lambda_{K}\right)} \mathcal{U}(\{\lambda\},-\{\lambda\})\right|_{\{\lambda\}=0} \\
& =\int_{-\infty}^{+\infty} \frac{d E}{2 \pi} \operatorname{Tr}\left[\boldsymbol{\Sigma}_{K}^{<}(E) \mathbf{G}^{>}(E)-\boldsymbol{\Sigma}_{K}^{>}(E) \mathbf{G}^{<}(E)\right] .
\end{aligned}
$$

Since no spin-flip events are allowed on the metalmolecule interface, the electronic self-energy due to coupling to the contacts is diagonal in spin space, that is,

$$
\Sigma_{\sigma}^{(K)}\left(\tau, \tau^{\prime}\right)=\sum_{k \in K}\left|V_{i k}\right|^{2} g_{k \sigma}\left(\tau, \tau^{\prime}\right),
$$

where $i=1$ for $K=L$ and $i=2$ for $K=R$, whereas $g_{k \sigma}$ is the Green function (GF) of a free electron. As a result, 
the charge current of electrons with spin $\sigma$ at interface $K$ is given by

$$
\begin{aligned}
I_{K \sigma}= & \frac{e}{\hbar} \int_{-\infty}^{+\infty} \frac{d E}{2 \pi} \\
& {\left[\Sigma_{\sigma}^{(K)<}(E) G_{i \sigma, i \sigma}^{>}(E)-\Sigma_{\sigma}^{(K)>}(E) G_{i \sigma, i \sigma}^{<}(E)\right] . }
\end{aligned}
$$

The spin and charge currents at interface $K$ are

$$
\begin{aligned}
& I_{K}^{(s)}=I_{K \uparrow}-I_{K \downarrow}, \\
& I_{K}^{(c)}=I_{K \uparrow}+I_{K \downarrow},
\end{aligned}
$$

respectively.

The second cumulant yields an expression for the zerofrequency noise. In the non-interacting model, it reads $\stackrel{41}{4}$

$$
\begin{aligned}
S_{K_{2} K_{1}}(\omega=0) & =-\left.i \frac{\partial}{\partial\left(i \lambda_{K_{2}}\right)} \frac{\partial}{\partial\left(i \lambda_{K_{1}}\right)} \mathcal{U}(\{\lambda\},-\{\lambda\})\right|_{\{\lambda\}=0} \\
=\int_{-\infty}^{+\infty} \frac{d E}{2 \pi} \operatorname{Tr} & \left\{\delta_{K_{1}, K_{2}}\left[\boldsymbol{\Sigma}_{K_{1}}^{<}(E) \mathbf{G}^{>}(E)+\mathbf{G}^{<}(E) \boldsymbol{\Sigma}_{K_{2}}^{>}(E)\right]-\mathbf{i}_{K_{1}}(E) \mathbf{i}_{K_{2}}(E)\right. \\
+ & \boldsymbol{\Sigma}_{K_{1}}^{<}(E) \mathbf{G}^{++}(E) \boldsymbol{\Sigma}_{K_{2}}^{>}(E) \mathbf{G}^{--}(E)+\boldsymbol{\Sigma}_{K_{1}}^{>}(E) \mathbf{G}^{--}(E) \boldsymbol{\Sigma}_{K_{2}}^{<}(E) \mathbf{G}^{++}(E) \\
& \left.-\boldsymbol{\Sigma}_{K_{1}}^{<}(E) \mathbf{G}^{>}(E) \boldsymbol{\Sigma}_{K_{2}}^{>}(E) \mathbf{G}^{<}(E)-\boldsymbol{\Sigma}_{K_{1}}^{>}(E) \mathbf{G}^{<}(E) \boldsymbol{\Sigma}_{K_{2}}^{<}(E) \mathbf{G}^{>}(E)\right\},
\end{aligned}
$$

where $\mathbf{i}_{K}(E)$ is a matrix of the energy-resolved current operator at interface $K$

$$
\mathbf{i}_{K}(E) \equiv \boldsymbol{\Sigma}_{K}^{<}(E) \mathbf{G}^{>}(E)-\boldsymbol{\Sigma}_{K}^{>}(E) \mathbf{G}^{<}(E)
$$

The spin and charge noise at interface $K$ are expressed by

$$
\begin{gathered}
S_{K K}^{(s)}=S_{K \uparrow, K \uparrow}-2 S_{K \uparrow, K \downarrow}+S_{K_{\downarrow}, K \downarrow}, \\
S_{K K}^{(c)}=S_{K \uparrow, K \uparrow}+2 S_{K \uparrow, K \downarrow}+S_{K_{\downarrow}, K \downarrow},
\end{gathered}
$$

where the spin resolved elements of the noise $S_{K \sigma, K \sigma^{\prime}}$ can be obtained from (25) by generalizing the counting field to include spin.

Higher order cumulants for a multi-level molecule can be similarly derived from (17) in a, cumbersome but, straightforward manner.

\section{HEATING}

Within the model (Fig. 1), the charge current is compensated at each interface so that only spins are transferred through the junction. Nevertheless, since electrons cross the molecule-contact interfaces also in the spin fieldeffect transistor, the question of heating of the molecular vibrations is still important. To estimate the temperature of the vibrations we extend an approximate scheme

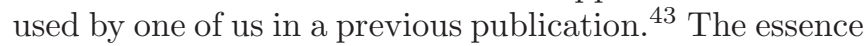
of the approach is quasi-particle assumption used to describe vibrational degrees of freedom (phonons). In this case the only relevant characteristic of the vibration (besides frequency) is its average population, while actual nonequilibrium distribution may be disregarded (density of states is a delta-function).

At steady-state influx of energy through the moleculecontact interfaces, $J^{\text {in }}$, is compensated by outflux, $J^{\text {out }}$, so that energy of the molecule does not change $J=J^{i n}-$ $J^{\text {out }}=0$. Since energy is carried by both electrons and phonons, we get

$$
J_{e}+J_{p h}=0 .
$$

Within the quasi-particle approximation the latter for a vibration $\omega_{i}$ coupled to a thermal bath is 45

$$
J_{p h}=\frac{\Omega\left(\omega_{i}\right)}{\hbar} \omega_{i}\left[N_{B E}\left(\omega_{i}\right)-N_{i}\right],
$$

where $\omega_{i}$ and $N_{i}(i=1,2)$ is a vibration and the corresponding average population of molecule $i$, respectively, whereas $\Omega$ is the spectral function of the bath, and $N_{B E}(\omega)=\left[e^{\beta \omega}-1\right]^{-1}$ is the Bose-Einstein distribution.

The electronic energy flux for a system coupled to a set of baths $B$ can be written as

$$
J_{e}=\sum_{B} \int_{-\infty}^{+\infty} \frac{d E}{2 \pi \hbar} E \operatorname{Tr}\left[\Sigma_{B}^{<}(E) G^{>}(E)-\Sigma_{B}^{>}(E) G^{<}(E)\right],
$$

where $\Sigma_{B}$ is the electronic self-energy due to the coupling to bath $B$. In our model we want to estimate the temperature of two vibrations, thus, following the approximate procedure introduced in Ref. 44, we split the system into 
two parts at the molecule-molecule interface. In this way we can speak about electronic energy flux in two parts of our system. Each part has two interfaces: one between the molecule and corresponding contact and the other between the molecules. The electronic self-energy due to the coupling to the contact is given by the standard expression (21). The self-energy on the molecule-molecule interface is given by (10).

We obtain the electronic flux in the molecule $i$ part of the system through the following procedure. We begin with the Hamiltonian $\hat{\bar{H}}$ which is transformed to the rotating reference frames of the magnetic fields, Eq.(5), however, without performing the small polaron transformation. We, then, treat the electron-vibration coupling in the Born approximation ${ }^{28}$. Within the non-crossing and quasi-particle approximations we accordingly obtain the electronic flux

$$
J_{e}=\omega_{i} M_{i}^{2}\left(\left[N_{i}+1\right] I_{i}^{(-)}-N_{i} I_{i}^{(+)}\right)
$$

where

$$
I_{i}^{( \pm)} \equiv \sum_{\sigma_{1}, \sigma_{2}} \int_{-\infty}^{+\infty} \frac{d E}{2 \pi} G_{\sigma_{1} \sigma_{2}}^{<}(E) G_{\sigma_{1} \sigma_{2}}^{>}\left(E \pm \omega_{i}\right)
$$

Using (30) and (32) in (29) yields

$$
N_{i}=\frac{\Omega\left(\omega_{i}\right) N_{B E}\left(\omega_{i}\right)-M_{i}^{2} I_{i}^{(-)}}{\Omega\left(\omega_{i}\right)+M_{i}^{2}\left(I_{i}^{(+)}-I_{i}^{(-)}\right)}
$$

The vibrational temperature is obtained from (34) under assumption that the vibrations are populated according to the Bose-Einstein distribution. We note that a more physically motivated procedure to introduce the temperature of the molecular vibrations at non-equilibrium can be used, $\stackrel{45}{=}$ however, for demonstration purposes the simpler procedure described above suffices.

Atomic cooling caused by sub-resonance optical excitation is a well-known effect. $\underline{46}$ In molecular junction the analogous detuning may lead to cooling of the molecular vibration. Here, we utilize the sub-resonance detuning of the electric and magnetic field frequencies from the energy difference of the inter-molecular and contactmolecule electronic transitions as a mechanism to pump energy out of the molecular vibration. Naturally, cooling is most efficient when the detuning frequency coincides with the frequency of the vibration. Additional possibilities to cool the current carrying molecular junction are discussed in Ref. 47 .

\section{NUMERICAL RESULTS}

Here we present numerical results in order to illustrate the transport properties of our model for the spin fieldeffect transistor sketched in Fig. 1. We use the level width $\Gamma$ due to coupling to a contact as unit of energy. Unless explicitly specified otherwise, the parameters for
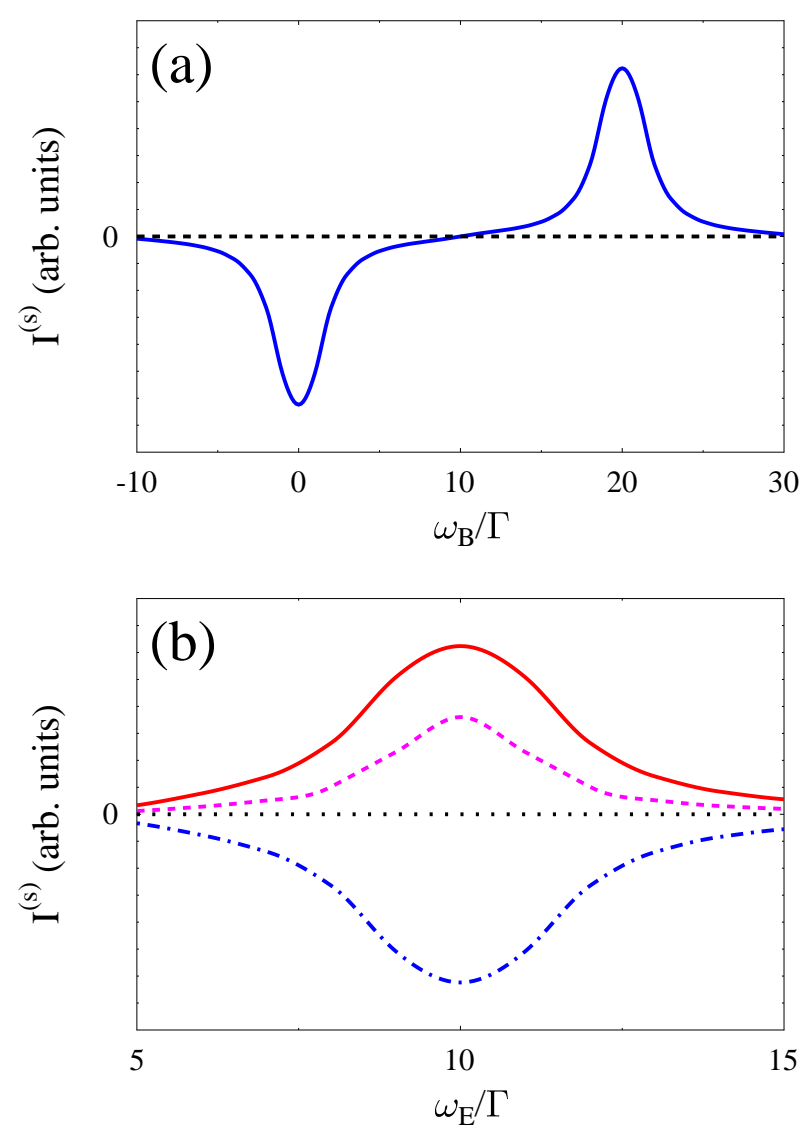

FIG. 2: (Color online) Elastic transport. Spin current vs. (a) $a c$ magnetic field frequency $\omega_{B}$ (solid line, blue) and (b) electric field frequency $\omega_{E}$. The latter shows results for $V_{E}=$ 0 (dotted line, black), 0.3 (dashed line, magenta), and 0.5 (solid line, red) at $\omega_{B}=20$. Also shown curve for $V_{E}=0.5$ at $\omega_{B}=0$ (dash-dotted line, blue). Dashed line (black) in (a) shows charge current. See text for other parameters.

calculations are temperature in the leads $T_{e}=0.3$, the positions of the levels in absence of external fields $\varepsilon_{1}=\varepsilon_{2}=0$, escape rates to the contacts (wide-band approximation is employed) $\Gamma_{\sigma}^{(K)}=1$, strength of coupling to external $d c$ magnetic field $\omega_{0} \equiv g \mu_{B} B^{(d c)}=-10$, strength of the coupling to the external $a c$ magnetic field $g \mu_{B} B^{(a c)}=0.01$ and its frequency $\omega_{B}=20$, strength of the coupling to the external electric field $V_{E}=0.5$. The parameters for the molecular vibrations are indicated in each case separately.

Figure 2 shows the spin current $I^{(s)}$ in the elastic transport regime. In the calculations, the shift of the levels due to changes in $\omega_{B}$ is assumed to be compensated by the $d c$ magnetic field, so that levels are set as in Fig. 11 and do not move. The spin current (solid line) vs. the external ac field frequency (spin bias) is plotted in Fig. 2a. Note that the electric field is an additional source of energy in the model, so that one will observe spin current even 

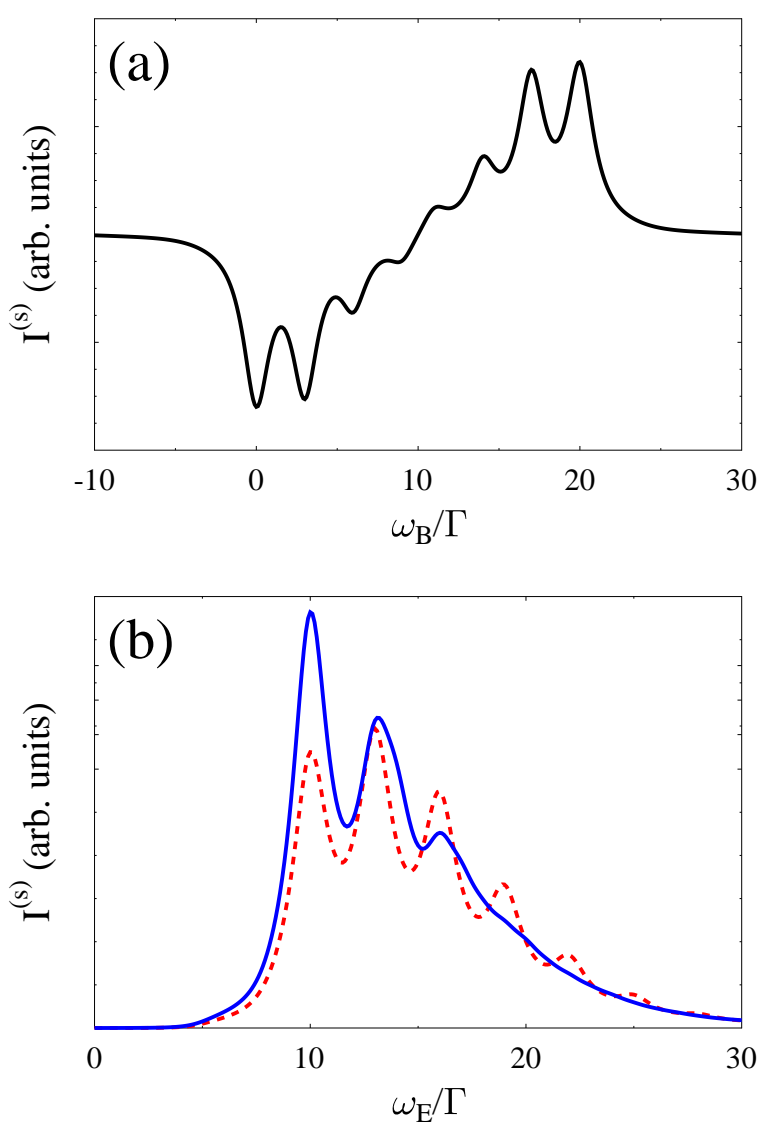

FIG. 3: (Color online) Inelastic transport. Spin current vs. (a) $a c$ magnetic field frequency $\omega_{B}$ and (b) electric field frequency $\omega_{E}$. The latter shows results for symmetric $\omega_{1}=\omega_{2}=3$ (dashed line, red) and asymmetric $\omega_{1}=3$, $\omega_{2}=4$ (solid line, blue) cases. Electron-vibration coupling is $M_{1}=M_{2}=3$. Other parameters are as in Fig. 2.

without spin bias in the contacts. The sign of the spin current shows the direction of the spin-up flux (direction from left to right is chosen as positive). The flux switches around $\omega_{B} / \Gamma=10$, as is shown in Fig. 2a. The point of sign change is defined by a competition between the spin bias and the electric field mediated inter-molecular transfer. At $\omega_{B}=0$ the lower spin levels are occupied, while the higher levels are empty (see Fig. 11). In this regime the electric field facilitates the spin-up flux from right to left, which defines a negative sign of the spin flux. The spin bias grows with the frequency $\omega_{B}$, and the value $\omega_{B} / \Gamma=10$ the two processes cancel each other. When the spin bias grows further, the population of the levels changes. The higher levels become populated due to the increased bias, while the population of lower levels diminishes (the level goes above the corresponding spin-resolved chemical potential, see Fig. 11). The positions of the peaks are defined by the resonance condition for inter-molecule electron transfer: the position of the molecular levels is kept fixed by the $d c$ field adjust- ment, while the frequency of the transition changes with both electric field frequency $\omega_{E}$ and $a c$ magnetic field frequency $\omega_{B}$, see Eq. (91). The condition for the resonance is $\omega_{E} \pm \omega_{B}=\left|\bar{\varepsilon}_{1 \sigma}-\bar{\varepsilon}_{2 \sigma}\right|$. We will return to the question of the role the spin bias plays in our discussion of the noise and heating properties. Note also, that the charge current (dashed line in Fig. 2a) is identically zero (the flux of spin up electrons from left to right is compensated by the flux of spin down electrons from right to left).

Fig. $2 \mathrm{~b}$ shows the dependence of the spin current on frequency of the external electric field. Naturally, the dependence has a maximum at resonance. Shown are plots for magnetic field frequency $\omega_{B}$ in the first (dashdotted line) and second (solid line) maximum of Fig. 2 2 a. Also shown are curves for smaller (dashed line) and zero coupling to the electric field (dotted). In the absence of coupling no spin current is observed. This indicates that, for the parameter range chosen, we are not in the regime of the spin pump described in Ref. 18 .

Figure 3 is inelastic analog of results presented in Fig. 2. Molecular vibrations are taken into account employing small polaron transformation, and assuming separation of timescales, so that introduction of the FranckCondon factors becomes possible. Fig. 3a shows spin current vs. frequency of $a c$ magnetic field. Parameters for molecular vibrations are $\omega_{1}=\omega_{2}=2$, electron-vibration coupling is $M_{1}=M_{2}=2$. Other parameters are as in Fig. 2. An unusual form of the vibration sidebands is caused by resonance condition for intermolecular electron transfer rather than resonance situation at moleculecontact interface. This makes vibrational characteristic different from the one presented in Ref. 20. At the same time, vibrational structure observed in spin current vs. electric field frequency resembles such for charge current vs. bias plots. Figure 3b shows two such characteristics: for symmetric $\omega_{1}=\omega_{2}=3$ (dashed line) and asymmetric $\omega_{1}<\omega_{2}\left(\omega_{1}=3\right.$ and $\omega_{2}=4$, solid line) cases. Presence of a higher frequency naturally leads to observation of less vibrational sidebands.

Figure 4 displays the noise properties of the junction. We show the results for elastic transport only, leaving the study of inelastic noise properties for a future publication. Fig. 4 4 shows the spin (or charge) zero-frequency noise as function of the external electric field frequency. Note that while the charge current is identically zero, charge noise does exist. Shown are the results for two situations: the spin levels of each molecule are well resolved (dashed line, red) vs. essential overlap between the two (solid line, blue). The first case corresponds to a situation with well pronounced resonance behavior. In such situations, the transmission probability at resonance approaches unity, which leads to suppression of the noise $\stackrel{48}{\underline{40}}$ The latter case corresponds to a situation when the transmission probability is lower. In this case no noise suppression is observed at resonance. The effect is similar to the behavior of a molecular junction in symmetric vs. asymmetric couplings at the two sides of the junction $\underline{42}$ 

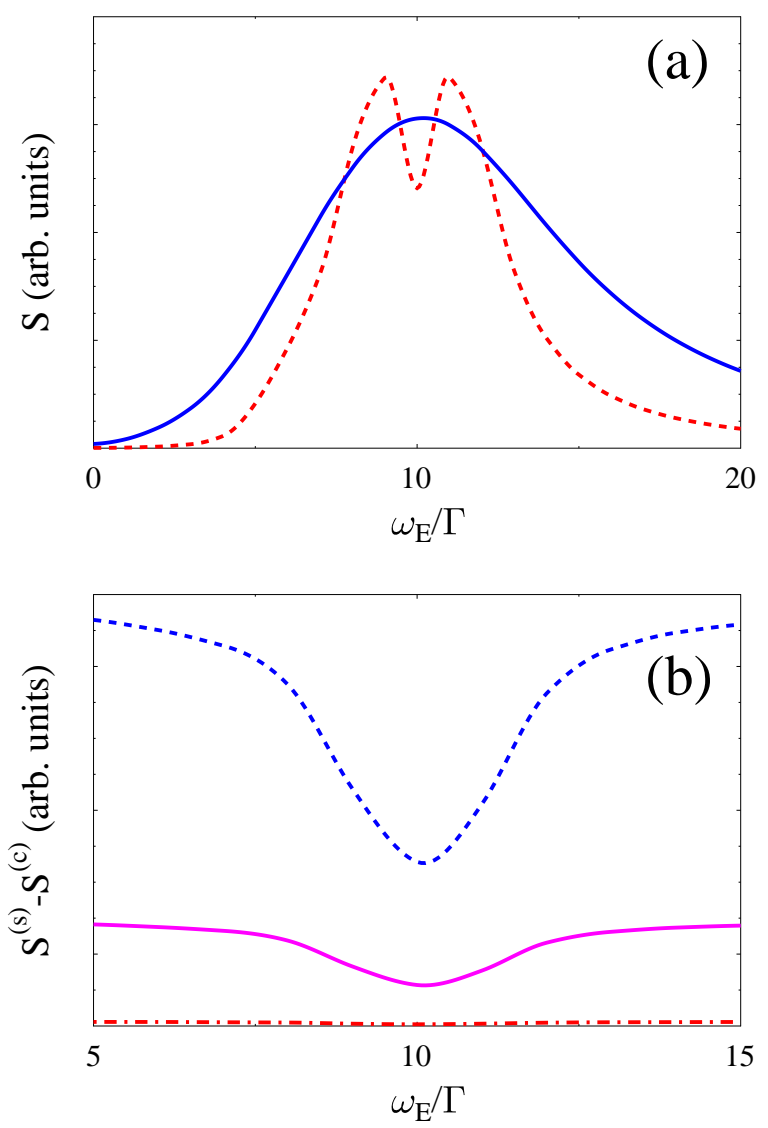

FIG. 4: (Color online) Noise properties. (a) Zero-frequency charge and spin noise for $\Gamma_{\sigma}^{(K)}=\Gamma$ (dashed line, red) and $\Gamma_{\sigma}^{(K)}=5$ (solid line, blue). vs. electric field frequency $\omega_{E}$. (b) Difference between spin and charge zero-frequency noise for $g \mu_{B} B^{(a c)}=0.01$ (dash-dotted line, red), 0.05 (solid line, magenta), and 0.1 (dashed line, blue). See text for parameters.

In contrast to charge noise, the spin noise strongly depends on spin-flip events within the system $\underline{\underline{49}}$ Fig. $4 \mathrm{~b}$ shows the difference between the spin and charge zerofrequency noise at several magnitudes of the coupling to the external ac magnetic field. Note that at resonance, where the electron transport through the junction becomes pronounced, the difference between the spin and charge components diminishes.

As was mentioned already earlier, there is no net charge transport between the leads. Nevertheless, charge transfer between contacts and molecule does occur. Thus, the spin field-effect transistor may be heated. As was discussed in the literature (see e.g. Refs. 47,50), charge transfer by itself does not necessarily lead to heating of the molecular device. Here, we demonstrate one such possibility. Cooling of a molecular vibration is caused by tuning the external field frequency out of resonance (an analog of atomic optical cooling). Figure 5 a
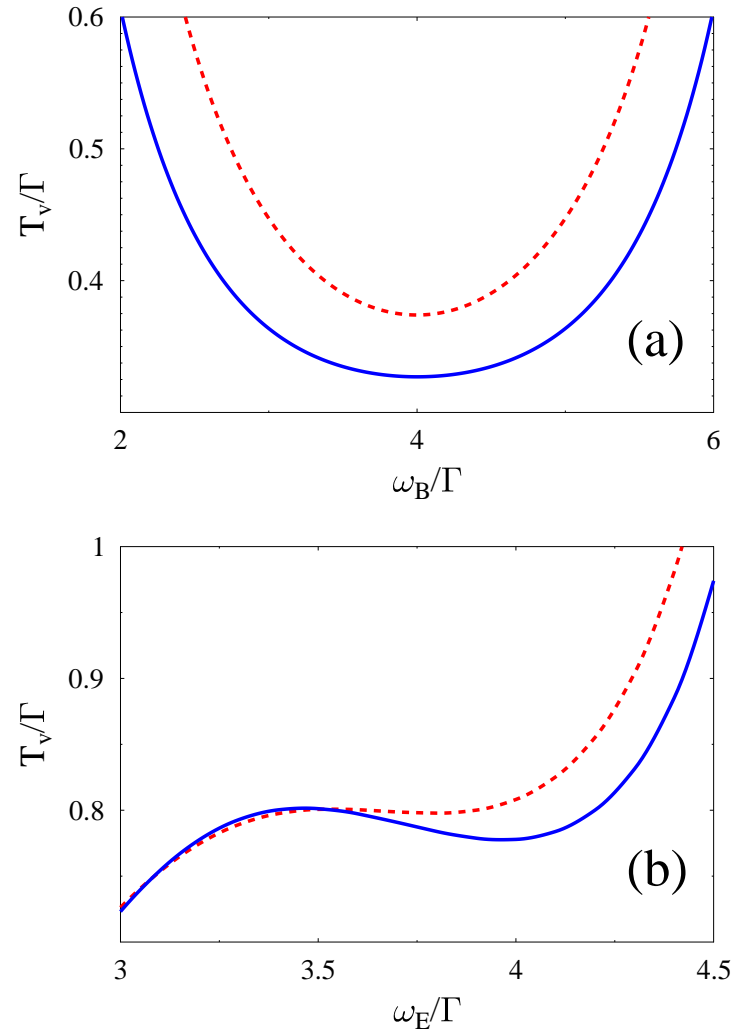

FIG. 5: (Color online) 'Temperature' of the first $\omega_{1}$ (dashed line, red) and second $\omega_{2}$ (solid line, blue) molecular vibration vs. (a) $a c$ magnetic field frequency $\omega_{B}$ and (b) external electric field frequency $\omega_{E}$. See text for parameters.

shows the temperatures of the first $\omega_{1}$ (dashed line) and second $\omega_{2}$ (solid line) molecular vibrations as functions of the spin bias. The coupling to the $d c$ magnetic field is $\omega_{0}=-4$, whereas the frequency of the electric field is $\omega_{E}=4$. The molecular vibrations are taken to be $\omega_{1}=1$ and $\omega_{2}=0.5$ with electron-vibration couplings $M_{1}=M_{2}=0.5$. Other parameters of the calculation are introduced at the start of the section. The parameters (electric and magnetic field frequencies) are chosen in such way that the most probable (resonant) electronic tunneling through the junction has to go uphill in energy at the contact-molecule and inter-molecule transition steps. In this case, the tunneling energy difference required for resonant electron transition is taken from the molecular vibration, which leads to cooling of the device. Fig. 5b shows similar behavior when the frequency of the electric field is used as a control instead. The molecular vibrations are $\omega_{1}=0.5$ and $\omega_{2}=0.2$. Here cooling takes place at the molecule-molecule interface only and is not as effective. However, one can achieve a stronger signal in this case. The efficiency of the cooling at maximum spin current is an interesting question for future studies. 


\section{CONCLUSION}

Spintronics is a quickly developing field of research (both experimental and theoretical). Here, we studied the spin current transport properties within a model for an all-electric controlled spin field-effect transistor. The model provides pure spin currents through a junction which consists of two spin-pumps (molecules under influence of external $d c$ magnetic fields ${ }^{18}$ each attached to its normal metal contact. The two parts are coupled by an external electric field, which serves as a control for the spin flux. Within a full counting statistics approach to multi-level systems, we discuss elastic and inelastic spin flux and noise properties of the junction. External electric and magnetic fields are indicated as possible controls of the spin current through the junction. The charge and spin zero-frequency noises are shown to be different when spin-flip processes within the junction become pronounced. Zero-frequency spin noise as function of the external electric field shows the same single to double peak structure transition when the tunneling probability approaches unity. Similar behavior was observed earlier for a model of charge field-effect transistor 42

Problem of molecular junction heating recently discussed in the literature in connection to charge transport, retains its importance also for spin molecular devices. Within the model, we discuss possibility to use external fields slightly de-tuned from the molecular resonances for cooling the molecular vibrations. This process is similar to optical cooling of atoms. We find that external $a c$ magnetic fields may be effective cooling media. External electric fields also provides the effect. The efficiency of the cooling at maximum spin flux is an interesting question for future studies.

\section{Acknowledgments}

J.F. thanks UCSD for its hospitality during his visit in July 2009, and the Swedish Research Council and the Royal Swedish Academy of Sciences for financial support. M.G. gratefully acknowledges support by the UCSD (startup funds), the UC Academic Senate (research grant), and the U.S.-Israel Binational Science Foundation.

\section{Appendix A: Derivation of Eq.(10)}

Here we derive an expression (10) for the self-energy due to the coupling to the external electric field within the rotating-wave approximation. We start by partitioning the total Hamiltonian of the system into a zero-order Hamiltonian $\hat{\bar{H}}_{0}$ and perturbation $\hat{\bar{V}}$, Eq. (9). The first is given by an analog of Eq.(2) after transformation to rotating frames. It will be similar to (2) with molecular and contact states energies renormalized according to (7) and (8), respectively, and without the time dependence of magnetic field terms. For the moment, we disregard the coupling to molecular vibrations. Hamiltonian $\hat{\bar{H}}_{0}$ defines the zero-order GFs of the system $\mathbf{G}^{(0)}$. We treat the interaction $\hat{\bar{V}}$ by perturbation theory, i.e expanding the evolution operator on the Keldysh contour (interaction representation) $\exp \left[-i \int_{c} d \tau \hat{V}_{I}(\tau)\right]$ to second order in $V_{E}$. This leads to a Dyson type equation for the Green function

$$
\begin{aligned}
& \mathbf{G}\left(\tau, \tau^{\prime}\right)=\mathbf{G}^{(0)}\left(\tau, \tau^{\prime}\right) \\
& +\int_{c} d \tau_{1} \int_{c} d \tau_{2} \mathbf{G}^{(0)}\left(\tau, \tau_{1}\right) \mathbf{\Sigma}^{(E)}\left(\tau_{1}, \tau_{2}\right) \mathbf{G}^{(0)}\left(\tau_{2}, \tau^{\prime}\right)
\end{aligned}
$$

with matrix elements for the self-energy given by

$$
\begin{aligned}
& \Sigma_{m \sigma_{1}, n \sigma_{2}}^{(E)}\left(\tau_{1}, \tau_{2}\right)=\delta_{m, n}\left(V_{E} e^{-i \omega_{E} t_{1}}+V_{E}^{*} e^{i \omega_{E} t_{1}}\right) \\
& \times\left(V_{E} e^{-i \omega_{E} t_{2}}+V_{E}^{*} e^{i \omega_{E} t_{2}}\right) \\
& \times G_{\bar{m} \sigma_{1}, \bar{m} \sigma_{2}}^{(0)}\left(\tau_{1}, \tau_{2}\right) \exp \left[i(-1)^{m} \omega_{B}\left(\sigma_{1} t_{1}-\sigma_{2} t_{2}\right)\right]
\end{aligned}
$$

where $m, n=1,2$ numerate molecule in the junction, whereas $\bar{m}$ means opposite of $m$. Application of the rotating wave approximation leaves only terms proportional to $\left|V_{E}\right|^{2}$ and enforces $\sigma_{1}=\sigma_{2}$, in (A2). The resulting expression is presented in Eq. (10).

In the case of the small polaron transformation, the coupling to molecular vibrations is treated by dressing the expression (10) by Franck-Condon factors

$$
\begin{aligned}
& \Sigma_{m \sigma, n \sigma^{\prime}}^{(E)}\left(\tau, \tau^{\prime}\right)=\delta_{m, n} \delta_{\sigma, \sigma^{\prime}} 2\left|V_{E}\right|^{2} \cos \omega_{E}\left(t-t^{\prime}\right) \\
& \times G_{\bar{m} \sigma, \bar{m} \sigma}\left(\tau, \tau^{\prime}\right) e^{i(-1)^{m} \sigma \omega_{B}\left(t-t^{\prime}\right)} \\
& \times\left\langle T_{c} \hat{X}_{\bar{m}}(\tau) \hat{X}_{\bar{m}}^{\dagger}\left(\tau^{\prime}\right)\right\rangle\left\langle T_{c} \hat{X}_{m}\left(\tau^{\prime}\right) \hat{X}_{m}^{\dagger}(\tau)\right\rangle
\end{aligned}
$$

When perturbation theory is employed, the GF in Eq. (A1) is considered as a zero-order GF of the Born approximation.
1 I.Žutić, J.Fabian, and S. Das Sarma. Rev. Mod. Phys. 76, 323 (2004).

2 R.Hanson, L.P.Kouwenhoven, J.R.Petta, S.Tarucha, and
L.M.K.Vandersypen. Rev. Mod. Phys. 79, 1217 (2007).

3 M.E.Flatté. Nature Physics 4, 587 (2008).

${ }^{4}$ M.Braun and G.Burkard. Phys. Rev. Lett. 101, 036802 
(2008).

5 J.J.Krich and B.I.Halperin. Phys. Rev. B 78, 035338 (2008)

6 S.Amasha, K.MacLean, I.P.Radu, D.M.Zumbuhl, M.A.Kastner, M.P.Hanson, and A.C.Gossard. Phys. Rev. Lett. 100, 046803 (2008).

7 P.Debray, S.M.S.Rahman, J.Wan, R.S.Newrock, M.Cahay, A.T.Ngo, S.E.Ulloa, S.T.Herbert, M.Muhammad, and M.Johnson. Nature Nanotechnology ASAP (2009).

8 J.Berezovsky, M.H.Mikkelsen, N.G.Stoltz, L.A.Coldren, and D.D.Awschalom. Science 320, 349 (2008).

9 F.Kuemmeth, S.Ilani, D.C.Ralph, and P.L.McEuen. Nature 452, 448 (2008).

10 D.Press, T.D.Ladd, B.Y.Zhang, and Y.Yamamoto Nature 456, 218 (2008).

11 J.R.Hauptmann, J.Paaske, and P.E.Lindelof. Nature Physics 4, 373 (2008).

12 C.Galland and A.Imamoglu. Phys. Rev. Lett. 101, 157404 (2008).

13 F.Dubin, M.Combescot, G.K.Brennen, R.Melet. Phys. Rev. Lett 101, 217403 (2008).

14 J.R.Petta, S.K.Slater, and D.C.Ralph. Phys. Rev. Lett. 93, 136601 (2004).

15 A.R.Rocha, V.M.Garcia-Suarez, S.W.Bailey, C.J.Lambert, J.Ferrer, and S.Sanvito. Nature Materials 4, 335 (2005).

16 S.Sanvito and A.R.Rocha. J. Comp. Theor. Nanoscience 3, 624 (2006).

17 L.Bogani and W.Wernsdorfer. Nature Materials 7, 179 (2008).

18 B.Wang, J.Wang, and H.Guo. Phys. Rev. B 67, 092408 (2003)

19 J.Peng and Z.Chen. Physics Lett. A 365, 505 (2007).

20 J.Fransson and J.-X.Zhu. Phys. Rev. B 78, 113307 (2008).

21 N.Jean and S.Sanvito. Phys. Rev. B 73, 094433 (2006).

22 J.Fransson. Nano Lett. 9, 2414 (2009).

23 B.Wang, J.Wang, and H.Guo. Phys. Rev. B 69, 153301 (2004).

24 H.A.Nilsson, P.Caroff, C.Thelander, M.Larsson, J.B.Wagner, L.-E.Wernersson, L.Samuelson, and H.Q.Xu. Nano Lett. 9, 3151 (2009).

25 P.Zhang, Q.-K.Xue, and X.C.Xie. Phys. Rev. Lett. 91, 196602 (2003).

26 G.D.Mahan. Many-Particle Physics. Kluwer Academic/Plenum Publishers (2000).

27 M.Galperin, A.Nitzan, and M.A.Ratner. Phys. Rev. B 76,
035301 (2007)

28 M.Galperin, M.A.Ratner, and A.Nitzan. J. Chem. Phys. 121, 11965 (2004).

29 L.P.Kadanoff and G.Baym. Quantum Statistical Mechanics W. A. Benjamin, Inc. (1962).

30 L.S.Levitov and G.B.Lesovik. JETP Lett. 58, 230 (1993).

31 L.S.Levitov, H.Lee, and G.B.Lesovik. J. Math. Phys. 37, 4845 (1996).

32 Yu.V.Nazarov. Ann. Phys. (Leipzig) 16, 720 (2007).

33 A.O.Gogolin and A.Komnik. Phys. Rev. B 73, 195301 (2006).

34 D.Djukic and J.M. van Ruitenbeek. Nano Lett. 6, 789 (2006).

35 T.L.Schmidt and A.Komnik. Phys. Rev. B 80, 041307(R) (2009).

36 R.Avriller and A. Levy Yeyati. Phys. Rev. B 80, 041309(R) (2009).

37 F.Haupt, T.Novotny, and W.Belzig. Phys. Rev. Lett. 103, 136601 (2009).

38 Note that Eq. (177) is equivalent to Eq.(1) of Ref. 37.

39 Y.Meir and N.S.Wingreen. Phys. Rev. Lett. 68, 2512 (1992).

40 H.Haug and A.-P.Jauho. Quantum Kinetics in Transport and Optics of Semiconductors. Springer (2008).

41 Deriving (25) from (17) we use mathematical matrix identity $\frac{d \mathbf{M}^{-1}(x)}{d x}=-\mathbf{M}^{-1}(x) \frac{d \mathbf{M}(x)}{d x} \mathbf{M}^{-1}(x)$

42 M.Galperin, A.Nitzan, and M.A.Ratner. Phys. Rev. B 74, 075326 (2006).

43 M.Galperin, M.A.Ratner, and A.Nitzan. J. Chem. Phys. 130, 144109 (2009).

44 M.Galperin and A.Nitzan. J. Chem. Phys. 124, 234709 (2006).

45 M.Galperin, A.Nitzan, and M.A.Ratner. Phys. Rev. B 75, 155312 (2007).

46 D.Leibfried, R.Blatt, C.Monroe, and D.Windeland. Rev. Mod. Phys. 75, 281 (2003).

47 M.Galperin, K.Saito, A.V.Balatsky, and A.Nitzan. Phys. Rev. B 80, 115427 (2009).

48 Y.M.Blanter and M.Buttiker. Physics Reports 336, 1 (2000).

49 M.Zareyan and W.Belzig. Europhys. Lett. 70, 817 (2005).

50 Z.Huang, F.Chen, R.DAgosta, P.A.Bennett, M. Di Ventra, and N. Tao, Nat. Nanotechnol. 2, 698 (2007). 